\title{
Conduction Shape Factor Models for Three-Dimensional Enclosures
}

\author{
P. Teertstra, ${ }^{*}$ M. M. Yovanovich, ${ }^{\dagger}$ and J. R. Culham ${ }^{\ddagger}$ \\ University of Waterloo, Waterloo, Ontario N2L 3G1, Canada
}

\begin{abstract}
Analytical models are presented for conduction shape factors for three-dimensional regions formed between an isothermal, arbitrarily shaped body and its concentric, arbitrarily shaped surrounding enclosure. The model is based on the exact solution for the concentric spheres, and two methods are developed to predict the effective gap spacing. The models are validated using existing numerical data from the literature and data from simulations performed using a commercial computational fluid dynamics software package. The models are shown to be in excellent agreement with the data for all enclosures with geometrically similar boundary shape, within $3 \% \mathrm{rms}$. For enclosures formed between different boundary shapes, the models are shown to be accurate within $5 \% \mathrm{rms}$ when the minimum aspect ratio, that is, the smallest outer boundary dimension over the largest inner dimension, is greater than 1.5 .
\end{abstract}

\begin{tabular}{ll} 
& \multicolumn{1}{c}{ Nomenclature } \\
$A$ & $=$ area, $\mathrm{m}^{2}$ \\
$a, b, c$ & $=$ cuboid side dimensions, $\mathrm{m}$ \\
$d$ & $=$ diameter, $\mathrm{m}$ \\
$k$ & $=$ thermal conductivity, $\mathrm{W} / \mathrm{mK}$ \\
$L$ & $=$ general characteristic length, $\mathrm{m}$ \\
$m$ & $=$ combination parameter \\
$\boldsymbol{n}$ & $=$ outward facing normal vector \\
$Q$ & $=$ total heat flow rate, $\mathrm{W}$ \\
$R$ & $=$ thermal resistance, $\mathrm{K} / \mathrm{W}$ \\
$\boldsymbol{r}$ & $=$ general radial coordinate \\
$S$ & $=$ conduction shape factor, $\mathrm{m}$ \\
$S_{L}^{\star}$ & $=$ dimensionless conduction shape factor, $S L / A_{i}$ \\
$S$ & $=$ cube side length, $\mathrm{m}$ \\
$T$ & $=$ temperature, ${ }^{\circ} \mathrm{C}$ \\
$V$ & $=$ enclosed volume, $\mathrm{m}^{3}$ \\
$x, y, z$ & $=$ Cartesian coordinates \\
$\delta$ & $=$ gap spacing, $\left(d_{o}-d_{i}\right) / 2, \mathrm{~m}$ \\
$\rho$ & $=$ local radial position, $\mathrm{m}$ \\
$\phi, \theta$ & $=$ spherical coordinates \\
$\psi$ & $=$ dimensionless temperature rise
\end{tabular}

Subscripts

$\begin{array}{ll}e & =\text { effective } \\ i & =\text { inner } \\ o & =\text { outer }\end{array}$

$\infty \quad=$ full-space limit

\section{Introduction}

A NALYTICAL models for conduction shape factors for threedimensional regions formed between an arbitrarily shaped,

Presented as Paper 2003-159 at the 41st Aerospace Sciences Meeting and Exhibit, Reno, NV, 6-9 January 2003; received 3 September 2004; revision received 3 December 2004; accepted for publication 4 December 2004. Copyright (C) 2005 by the American Institute of Aeronautics and Astronautics, Inc. All rights reserved. Copies of this paper may be made for personal or internal use, on condition that the copier pay the $\$ 10.00$ per-copy fee to the Copyright Clearance Center, Inc., 222 Rosewood Drive, Danvers, MA 01923; include the code 0887-8722/05 \$10.00 in correspondence with the CCC.

* Research Assistant Professor, Microelectronics Heat Transfer Laboratory, Department of Mechanical Engineering; pmt@mhtl.uwaterloo.ca.

Distinguished Professor Emeritus, Microelectronics Heat Transfer Laboratory, Department of Mechanical Engineering; mmyov@ mhtl.uwaterloo.ca. Fellow AIAA.

¥Associate Professor, Director Microelectronics Heat Transfer Laboratory, Department of Mechanical Engineering; rix@mhtl.uwaterloo.ca. heated body and its surrounding, cooled enclosure are of interest to thermal designers of sealed enclosures for microelectronics applications. Analytical heat transfer models can be used to predict quickly and accurately operating temperatures of circuit boards and components in the enclosure, providing a tool for parametric studies and tradeoff analyses during the preliminary design process. An important step in the formulation of natural convection models for these applications is the development of conduction models in the enclosed region.

The problem of interest in the current study involves steady-state conduction in the isotropic region bounded by concentric, arbitrarily shaped isothermal boundaries, as shown in Fig. 1. Geometries examined include enclosed regions between similar body shapes with constant or near-constant gap thickness, as well as enclosures with different inner and outer boundary shapes.

Numerical data are available in the literature for steady-state conduction for a limited set of enclosure geometries. Hassani ${ }^{1}$ presents data for the concentric circular cylinders and the concentric baseattached cones for a wide range of gap spacing. Warrington et al. ${ }^{2}$ present numerical data for enclosures formed between different concentric boundary shapes for two cases: the cube in a spherical enclosure and the sphere in a cubical enclosure.

The only analytical model for the conduction shape factor in the literature is presented by Hassani and Hollands ${ }^{3}$ for the dimensional shape factor $S$ for three-dimensional enclosure geometries with uniform gap spacing,

$$
S=\left(S_{0}^{m}+S_{\infty}^{m}\right)^{1 / m}
$$

where $S_{\infty}$ for the sphere is used to approximate all body shapes

$$
S_{\infty}=3.51 \sqrt{A_{i}}
$$

and $S_{0}$ is approximated based on one-dimensional heat transfer

$$
S_{0}=A_{i} / \delta
$$

where $\delta$ is the minimum value of the gap spacing. The blended combination in Eq. (1) reduces to linear superposition, $m=1$, for the concentric spheres. However, for boundary shapes where corners are present, such as the concentric cubes, a combination parameter value $m>1$ is introduced. The values for $m$ are dependent on geometry and are determined from a correlation of numerical data as a function of the inner body surface area, aspect ratio, and the enclosed volume. The model is restricted to enclosed regions formed between geometrically similar boundary shapes, such that the gap spacing is uniform. There are currently no analytical models available in the literature that address the problem of conduction for an enclosure with a nonuniform gap spacing. 
Fig. 1 Schematic of physical problem.

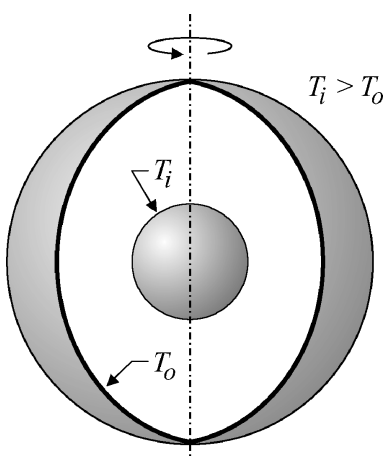

The objective of the current study is to develop analytical models for the conduction shape factor for the general case of enclosures formed between two arbitrarily shaped, isothermal boundaries. These models will be validated using the available numerical data from the literature, as well as data from a commercial computational fluid dynamics (CFD) package for a variety of different boundary shapes.

\section{Problem Definition}

The conduction shape factor $S$ is defined by Yovanovich ${ }^{4}$ for an isolated, three-dimensional body shape based on an area integral of the temperature gradient along an outward facing normal $n$ from the body surface,

$$
S=\iint_{A_{i}}-\left.\frac{\partial \psi}{\partial n}\right|_{A_{i}} \mathrm{~d} A_{i}
$$

For the particular problem of the three-dimensional enclosure formed between a heated inner and cooled outer boundary, the integral is evaluated at the inner surface $A_{i}$ where the dimensionless temperature rise $\psi$ is defined as

$$
\psi=\left[T(\boldsymbol{r})-T_{o}\right] /\left(T_{i}-T_{o}\right)
$$

The shape factor is nondimensionalized using the general scale length $L$, as follows:

$$
S_{L}^{\star}=\frac{S L}{A_{i}}=\frac{L}{A_{i}} \iint_{A_{i}}-\left.\frac{\partial \psi}{\partial n}\right|_{A_{i}} \mathrm{~d} A_{i}
$$

The dimensionless shape factor is related to the thermal resistance $R$ and total heat transfer rate $Q$ by

$$
S_{L}^{\star}=\frac{L}{k A_{i} R}=\frac{Q L}{k A_{i}\left(T_{i}-T_{o}\right)}
$$

The square root of the inner body surface area is selected as the characteristic length, $L=\sqrt{ } A_{i}$, such that

$$
S_{\sqrt{A_{i}}}^{\star}=1 / k \sqrt{A_{i}} R=Q /\left[k \sqrt{A_{i}}\left(T_{i}-T_{o}\right)\right]
$$

Figure 2 presents the range of different inner body shapes examined in this work and shows the notation used to describe each of the dimensions. Similar notation is used for the dimensions of the outer boundary shapes.

\section{Model Development}

The concentric spherical enclosure is a fundamental case of threedimensional enclosures, where the thermal resistance can be determined using the expression presented in most heat transfer texts, ${ }^{5}$

$$
R=(1 / 2 \pi k)\left(1 / d_{i}-1 / d_{o}\right)
$$

Using Eq. (6) to relate the resistance to the dimensionless conduction shape factor gives

$$
S_{\sqrt{A_{i}}}^{\star}=\frac{1}{k \sqrt{A_{i}} R}=\frac{2 \sqrt{\pi}}{\left(1-d_{i} / d_{o}\right)}
$$

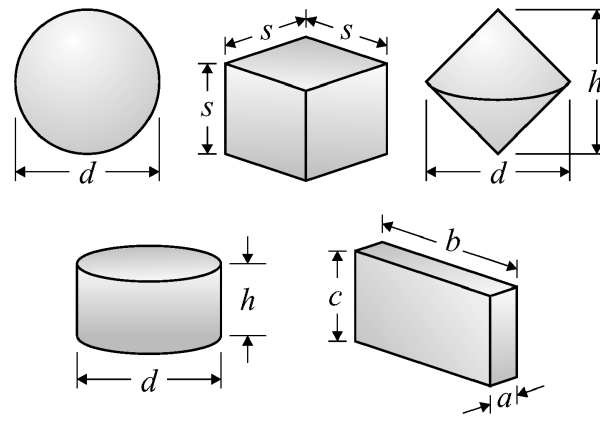

Fig. 2 Inner and outer boundary shapes.

However, the asymptotic characteristics of the shape factor solution for small and large values of aspect ratio $d_{i} / d_{o}$ can be demonstrated more effectively by introducing the gap thickness $\delta$, defined as

$$
\delta=\left(d_{o}-d_{i}\right) / 2
$$

Substituting $d_{o}=d_{i}+2 \delta$ into the expression for thermal resistance gives

$$
R=\delta / \pi k d_{i}\left(d_{i}+2 \delta\right)
$$

The corresponding dimensionless shape factor expression is

$$
\begin{aligned}
S_{\sqrt{A_{i}}}^{\star} & =1 / k \sqrt{A_{i}} R=1 / k \sqrt{\pi} d_{i} \cdot \pi k d_{i}\left(d_{i}+2 \delta\right) / \delta \\
& =\sqrt{\pi} d_{i} / \delta+2 \sqrt{\pi}
\end{aligned}
$$

This formulation for $S_{\sqrt{ } A_{i}}^{\star}$ is a linear superposition of two limiting cases. For small values of gap spacing with respect to the inner body diameter, $\delta / d_{i}$, the first term in Eq. (11) is dominant, corresponding to one-dimensional planar resistance. For large $\delta / d_{i}, S^{\star}{ }_{A_{i}}$ tends to a constant value corresponding to the dimensionless conduction shape factor for a sphere in a full-space region.

When it is noted that the numerator of the first term in Eq. (11) corresponds to $\sqrt{ } A_{i}$ for the sphere, a general model for dimensionless conduction shape factor for three-dimensional enclosures is proposed based on the exact solution for the concentric spheres,

$$
S_{\sqrt{A_{i}}}^{\star}=\left(\sqrt{A_{i}} / \delta_{e}\right)+S_{\infty}^{\star}
$$

where $S_{\infty}^{\star}$ is the conduction shape factor for the inner body in a full-space region. Models, tabulated values, and correlations for $S_{\infty}^{\star}$ are available for a variety of body shapes in technical publications or handbooks. ${ }^{4}$

\section{Effective Gap Spacing}

The effective gap spacing in Eq. (12), $\delta_{e}$, is an exact value in the case of enclosures with uniform gap thickness, such as the concentric spheres. However, for enclosures with different inner and outer boundary shapes or boundaries with sharp corners, approximate models are required to predict $\delta_{e}$. In the proposed research study, two different models for the effective gap spacing are developed.

For certain geometries, it is possible to calculate the local gap thickness as a function of the angular position in spherical coordinates, where the origin is concentric with the inner and outer boundaries. For the particular example of a cube in a spherical enclosure, local gap thickness $\delta(\phi, \theta)$ is determined by

$$
\delta(\phi, \theta)=\left(d_{o} / 2\right)-\rho_{i}(\phi, \theta)
$$

where $\rho_{i}(\phi, \theta)$ maps one-quarter of the $x=s_{i} / 2$ face of the cube in spherical coordinates, as shown in Fig. 3,

$$
\begin{gathered}
\rho_{i}(\phi, \theta)=\frac{\left(s_{i} / 2\right)}{\sin \phi \cos \theta} \\
0 \leq \theta \leq \frac{\pi}{4}, \quad \tan ^{-1} \sec \theta \leq \phi \leq \frac{\pi}{2}
\end{gathered}
$$




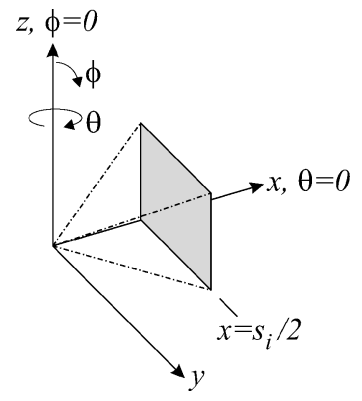

Fig. 3 Cube surface definition in spherical coordinates.

The mean value for the effective gap spacing is determined by an area integration in spherical coordinates over the outer boundary $A_{\circ}$ :

$$
\delta_{e}=\frac{24}{\pi d_{o}^{2}} \int_{0}^{\pi / 4} \int_{\tan ^{-1} \sec \theta}^{\pi / 2} \delta(\phi, \theta) \frac{d_{o}^{2}}{4} \sin \phi \mathrm{d} \phi \mathrm{d} \theta
$$

The integral is solved numerically to determine $\delta_{e}$ for different combinations of $d_{o}$ and $s_{i}$.

For certain combinations of boundary shapes, it may be difficult to define the local gap thickness as a mathematical function that can be integrated in the manner described. In this case, an alternate approximate modeling technique called the two-rule method has been developed by the authors. In this method, the effective gap spacing is determined from the equivalent spherical enclosure in which the surface area of the inner boundary and the volume of the enclosed region are preserved. The diameter of the equivalent inner sphere is found by

$$
d_{i}=\sqrt{A_{i} / \pi}
$$

The diameter of the equivalent outer spherical boundary is related to the volume of the inner body $V_{i}$ and the total enclosed volume $V$ by

$$
d_{o}=\left[6\left(V+V_{i}\right) / \pi\right]^{\frac{1}{3}}
$$

The volume of the inner sphere is related to its area by

$$
V_{i}=(\pi / 6) d_{i}^{3}=A_{i}^{\frac{3}{2}} / 6 \sqrt{\pi}
$$

The effective gap spacing is determined by

$$
\begin{aligned}
\delta_{e}= & \frac{d_{o}-d_{i}}{2}=\frac{1}{2}\left[\left(\frac{6 V}{\pi}+\frac{A_{i}^{\frac{3}{2}}}{\pi^{\frac{3}{2}}}\right)^{\frac{1}{3}}-\left(\frac{A_{i}}{\pi}\right)^{\frac{1}{2}}\right] \\
= & \frac{\sqrt{A_{i}}}{2 \sqrt{\pi}}\left[\left(6 \sqrt{\pi} \frac{V}{A_{i}^{\frac{3}{2}}}+1\right)^{\frac{1}{3}}-1\right]
\end{aligned}
$$

\section{Conduction Shape Factor}

Given these two methods for calculating the effective gap spacing, the conduction shape factor can be determined based on Eq. (12). For the integral method, the conduction shape factor is

$$
S_{\sqrt{A_{i}}}^{\star}=\left(\sqrt{A_{i}} / \delta_{e}\right)+S_{\infty}^{\star}
$$

where $\delta_{e}$ is determined based on a numerical solution to the integral, where necessary. In the case of the two-rule model, $\delta_{e}$ from Eq. (19) can be substituted into Eq. (12) to yield

$$
S_{\sqrt{A_{i}}}^{\star}=\frac{2 \sqrt{\pi}}{\left[1+6 \sqrt{\pi}\left(V^{\frac{1}{3}} / \sqrt{A_{i}}\right)^{3}\right]^{\frac{1}{3}}-1}+S_{\infty}^{\star}
$$

where the dimensionless independent parameter $V^{1 / 3} / \sqrt{ } A_{i}$ is selected to match the order of the terms appearing in the general model, Eq. (12). In cases involving geometrically similar boundary shapes, such as the concentric cubes or cylinders, the lower limit on the independent parameter is $V^{1 / 3} / \sqrt{ } A_{i} \rightarrow 0$, where the conduction shape factor approaches infinity as the inner and outer boundaries come into contact. However, when the inner and outer boundary shapes are different, the lower limit on $V^{1 / 3} / \sqrt{ } A_{i}$ is a finite, nonzero value. For the example of a sphere in a cubical enclosure when the diameter of the inner sphere $d_{i}$ equals the side length of the cube $s_{o}$, the total enclosed volume and independent parameter values are

$$
V=s_{o}^{3}-(\pi / 6) d_{i}^{3} \approx 0.476 s_{o}^{3}, \quad V^{\frac{1}{3}} / \sqrt{A_{i}} \approx 0.441
$$

As a result, the limit of $S_{\sqrt{ } A_{i}}^{\star} \rightarrow \infty$ occurs when $V^{\frac{1}{3}} / \sqrt{ } A_{i} \rightarrow 0.441$ for the sphere in a cubical enclosure.

\section{Model Application and Validation}

To validate the conduction shape factor models for a variety of inner and outer boundary configurations, the following test cases are presented.

\section{Geometrically Similar Boundary Shapes}

Three different configurations of enclosures with geometrically similar boundaries are examined: concentric cubes, concentric circular cylinders, and concentric base-attached double cones. For each configuration, the ratio of the inner to outer boundary dimensions are varied such that the range of the independent parameter is

$$
0.2 \leq V^{\frac{1}{3}} / \sqrt{A_{i}} \leq 5
$$

The conduction shape factor $S^{\star}{ }_{J_{i}}$ is determined using the two-rule model, Eq. (21), as a function of the boundary dimensions defined in Fig. 2.

Concentric Cubes

$$
\begin{gathered}
V=s_{o}^{3}-s_{i}^{3}, \quad A_{i}=6 s_{i}^{2} \\
S_{\sqrt{A_{i}}}^{\star}=\frac{2 \sqrt{\pi}}{\left\{1+(\sqrt{\pi} / 6)\left[\left(s_{o} / s_{i}\right)^{3}-1\right]\right\}^{\frac{1}{3}}-1}+S_{\infty}^{\star}
\end{gathered}
$$

where the conduction shape factor for the isolated cube in a fullspace domain is ${ }^{4}$

$$
S_{\infty}^{\star}=3.391
$$

Concentric Circular Cylinders, $\mathrm{h} / \mathrm{d}=1$

$$
\begin{gathered}
V=\frac{\pi}{4}\left(d_{o}^{2} h_{o}-d_{i}^{2} h_{i}\right), \quad A_{i}=2\left(\frac{\pi}{4} d_{i}^{2}\right)+\pi d_{i} h_{i} \\
S_{\sqrt{A_{i}}}^{\star}=\frac{2 \sqrt{\pi}}{\left\{1+(2 / \sqrt{6})\left[\left(d_{o} / d_{i}\right)^{3}-1\right]\right\}^{\frac{1}{3}}-1}+S_{\infty}^{\star}
\end{gathered}
$$

where $S_{\infty}^{\star}$ is calculated by ${ }^{4}$

$$
S_{\infty}^{\star}=\frac{3.1915+2.7726\left(h_{i} / d_{i}\right)^{0.76}}{\sqrt{1+2 h_{i} / d_{i}}}=3.443
$$

Concentric Base-Attached, Double Cones, $\mathrm{h} / \mathrm{d}=1$

$$
\begin{aligned}
& V=\frac{\pi}{12}\left(d_{o}^{2} h_{o}-d_{i}^{2} h_{i}\right), \quad A_{i}=2\left[\frac{\pi}{4} d_{i} h_{i}\right] \\
& S_{\sqrt{A_{i}}}^{\star}=\frac{2 \sqrt{\pi}}{\left\{1+\sqrt{2}\left[\left(d_{o} / d_{i}\right)^{3}-1\right]\right\}^{\frac{1}{3}}-1}+S_{\infty}^{\star}
\end{aligned}
$$




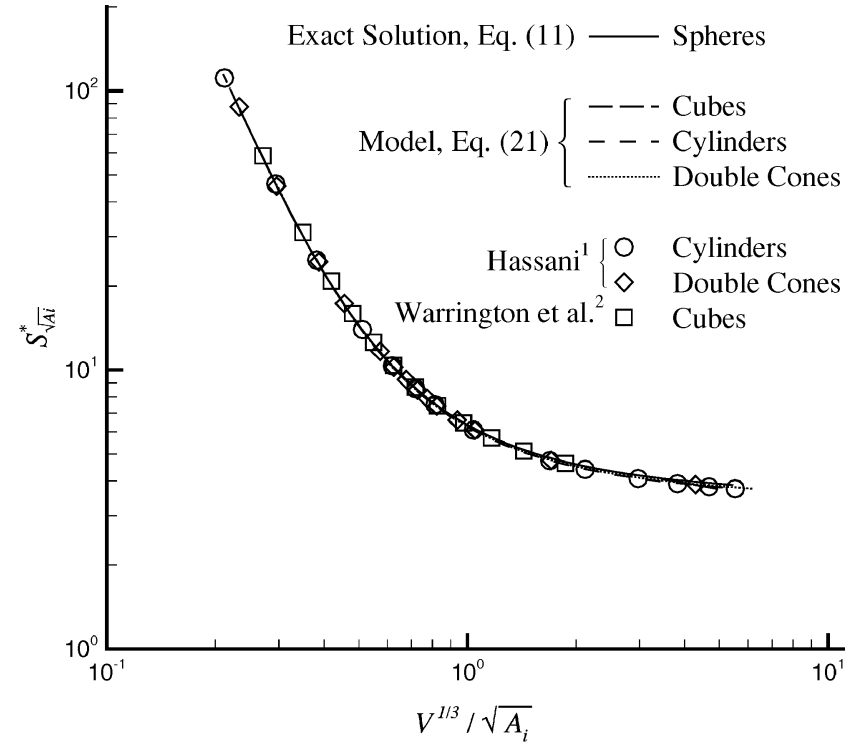

Fig. 4 Geometrically similar boundary shapes.

where $S_{\infty}^{\star}$ is determined from the correlation, ${ }^{4}$

$$
\begin{aligned}
S_{\infty}^{\star}= & 3.1943+0.6266 \frac{h_{i}}{d_{i}}-0.4778{\frac{h_{i}{ }^{2}}{d_{i}}} \\
& +0.0751{\frac{h_{i}{ }^{3}}{d_{i}}}^{3}+0.0532{\frac{h_{i}}{d_{i}}}^{4}=3.471
\end{aligned}
$$

In Fig. 4, the model predictions for each of these enclosure configurations are compared with numerical data from Hassani ${ }^{1}$ for the cylinders and base-attached double cones and from Warrington et al. ${ }^{2}$ for the concentric cubes, as well as for the exact solution for the concentric spheres. Figure 4 demonstrates the excellent agreement between the two-rule model and the data, with an rms difference of $2.8 \%$ for the concentric cubes and a difference of less than $1 \%$ rms for both the cylinders and double cones. The use of $V^{1 / 3} / \sqrt{ } A_{i}$ as the independent parameter and the square root of the inner body surface area as the scale length has effectively collapsed all of the data and models to a single curve.

\section{Cube in Spherical Enclosure}

The conduction shape factor for the region formed between a cube and a concentric, spherical enclosure is modeled using both the two-rule and integral methods. The ratio of the inner to the outer boundary dimensions are varied over a range corresponding to the available data from Warrington et al., ${ }^{2}$

$$
0.5 \leq V^{\frac{1}{3}} / \sqrt{A_{i}} \leq 2
$$

When the two-rule model is used, the effective gap spacing $\delta_{e}$ and the dimensionless conduction shape factor $S_{{ }^{\star} A_{i}}$ are determined using Eqs. (19) and (20),

$$
\begin{gathered}
V=\frac{\pi}{6} d_{o}^{3}-s_{i}^{3}, \quad A_{i}=6 s_{i}^{2} \\
\delta_{e}=\frac{s_{i}}{2}\left\{\left[\left(\frac{d_{o}}{s_{i}}\right)^{3}+\frac{6(\sqrt{6}-\sqrt{\pi})}{\pi \sqrt{\pi}}\right]^{\frac{1}{3}}-\sqrt{6 / \pi}\right\} \\
S_{\sqrt{A_{i}}}^{\star}=\frac{2 \sqrt{\pi}}{\left\{1+(\pi \sqrt{\pi} / 6 \sqrt{6})\left[\left(d_{o} / s_{i}\right)^{3}-6 / \pi\right]\right\}^{\frac{1}{3}}-1}+3.391
\end{gathered}
$$

Because the geometry of both the spherical and cubical boundaries can be expressed in spherical coordinates, the integral method can also be used in this case. As described by Eqs. (13) and (14), the local gap thickness can be determined as a function of angular position, and the effective gap spacing is determined from an area

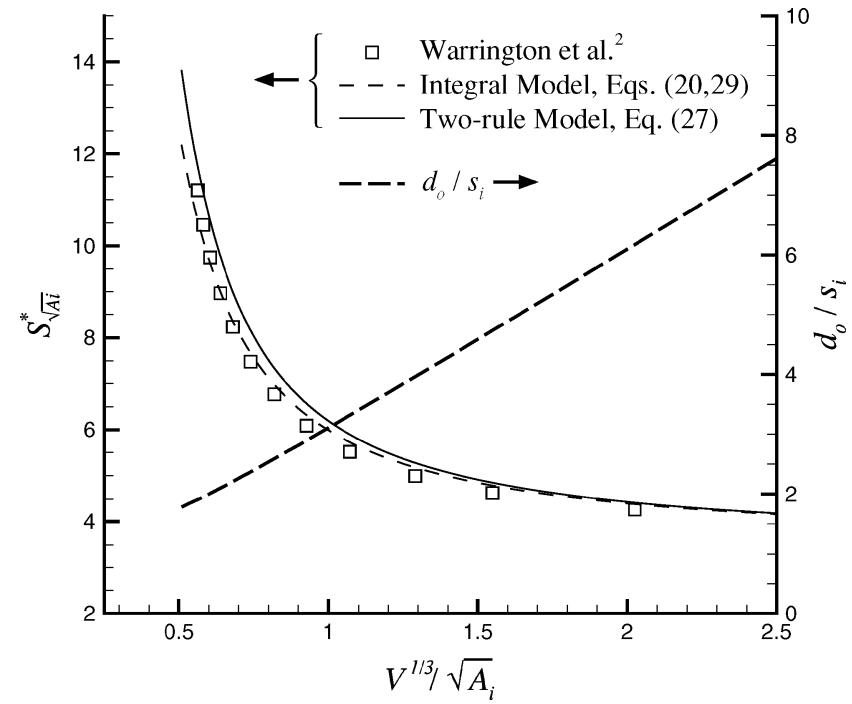

Fig. 5 Cube in spherical enclosure.

average over the outer, spherical boundary. The integral shown in Eq. (15) can be partially evaluated to the following expression:

$$
\delta_{e}=\frac{s_{i}}{2}\left[\frac{d_{o}}{s_{i}}-3 \ln (1+\sqrt{2})\right]+\frac{3 s_{i}}{\pi} \int_{0}^{\pi / 4} \frac{\tan ^{-1} \sec \theta}{\cos \theta} \mathrm{d} \theta
$$

which can be evaluated numerically to give

$$
\delta_{e}=s_{i}\left[\frac{1}{2}\left(d_{o} / s_{i}\right)-0.6107\right]
$$

The conduction shape factor for the integral method is determined by substituting Eq. (30) into the general model for $S_{\sqrt{ } A_{i}}^{\star}$, Eq. (20).

Figure 5 compares the predictions of the integral and two-rule models with numerical data from Warrington et al. ${ }^{2}$ The ratio of the diameter of the spherical enclosure and the cube side length, $d_{o} / s_{i}$, is also plotted vs $V^{1 / 3} / \sqrt{ } A_{i}$ on the second $y$ axis. There is good agreement between both models and the data; however, the integral model provides a better fit of the data than the two-rule model, within $3 \% \mathrm{rms}$ difference over the full range of the dimensionless gap spacing.

\section{Sphere in Cubical Enclosure}

For the sphere contained with a concentric cubical enclosure, both the two-rule and integral methods for predicting $\delta_{e}$ and $S^{\star}{ }_{A_{i}}$ are compared with the existing numerical data. Applying the tworule model to this case gives the following:

$$
\begin{gathered}
V=s_{o}^{3}-\frac{\pi}{6} d_{i}^{3}, \quad A_{i}=\pi d_{i}^{2} \\
\delta_{e}=\frac{d_{i}}{2}\left[\left(\frac{6}{\pi}\right)^{\frac{1}{3}}\left(\frac{s_{o}}{d_{i}}\right)-1\right] \\
S_{\sqrt{A_{i}}}^{\star}=\frac{2 \sqrt{\pi}}{\left[(6 / \pi)^{\frac{1}{3}} s_{o} / d_{i}-1\right]}+2 \sqrt{\pi}
\end{gathered}
$$

For the integral method analysis, the effective gap spacing is determined based on an area average on the inner spherical surface by interchanging $d_{o}$ with $d_{i}$ in Eq. (15), where $\delta(\phi, \theta)$ is

$$
\begin{gathered}
\delta(\phi, \theta)=\frac{s_{o} / 2}{\sin \phi \cos \theta}-\frac{d_{i}}{2}, \quad 0 \leq \theta \leq \frac{\pi}{4} \\
\tan ^{-1} \sec \theta \leq \phi \leq \frac{\pi}{2}
\end{gathered}
$$

Partial evaluation of the integral gives

$$
\delta_{e}=\frac{3 s_{o}}{2} \ln (1+\sqrt{2})-\frac{3 s_{o}}{\pi} \int_{0}^{\pi / 4} \frac{\tan ^{-1} \sec \theta}{\cos \theta} \mathrm{d} \theta-\frac{d_{i}}{2}
$$




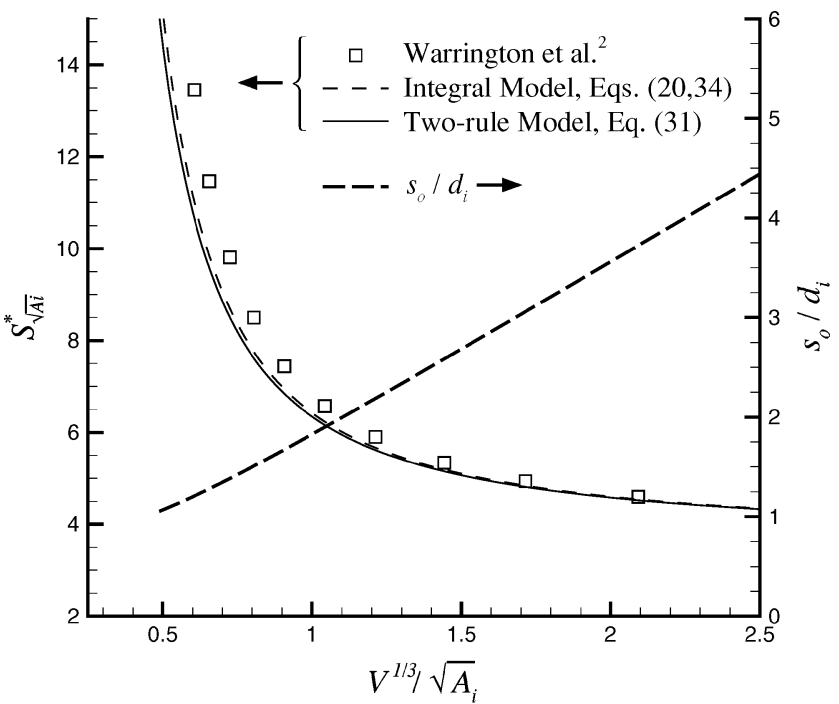

Fig. 6 Sphere in cubical enclosure.

Evaluating the integral numerically yields

$$
\delta_{e}=d_{i}\left[0.6107\left(s_{o} / d_{i}\right)-\frac{1}{2}\right]
$$

Figure 6 compares the $S_{\sqrt{ } A_{i}}^{\star}$ data of Warrington et al. ${ }^{2}$ with both the two-rule and integral models, as well as the ratio $s_{o} / d_{i}$, as a function of $V^{1 / 3} / \sqrt{ } A_{i}$. The agreement between the models and data is not as good as for the cube in a spherical enclosure; however, for $V^{1 / 3} \sqrt{ } A_{i}>1$, corresponding to $s_{o} / d_{i}>2$, the rms difference between both models and the data is less than $5 \%$. The error becomes more significant for smaller gap spacing, where the diameter of the inner sphere approaches that of the outer boundary, leading to a local increase in heat transfer that is not accounted for in the model.

\section{Cuboid in Cubical Enclosure}

In the next test case, the conduction shape factor for a cuboid with dimensions $a, b=3.785 a$, and $c=2.175 a$ in a concentric cubical enclosure is modeled. Because of the complexity of formulating the limits on the integration for each of the faces of the cuboid, the integral method will not be used in this example. The two-rule model is applied as follows:

$$
\begin{array}{r}
V=s_{o}^{3}-a b c, \quad A_{i}=2 a b+2 a c+2 b c \\
\frac{V^{\frac{1}{3}}}{\sqrt{A_{i}}}=\frac{\left(s_{o}^{3} / a b c-1\right)^{\frac{1}{3}}}{\sqrt{2(a b c)^{\frac{1}{3}}(1 / a+1 / b+1 / c)}}
\end{array}
$$

$S_{\sqrt{ } A_{i}}^{\star}$ is calculated using Eq. (20), where $S_{\infty}^{\star}$ is calculated using the model presented by Yovanovich ${ }^{4}$ and Culham et al. ${ }^{6}$ for the cuboid, which gives $S_{\infty}^{\star}=3.469$.

The model is compared to numerical data from simulations performed using FLOTHERM, ${ }^{7}$ a commercial finite volume based CFD package. Full details of the numerical procedure, including a grid convergence study, are given in the Appendix. The predictions of the two-rule model are compared with the numerical data in Fig. 7. As in the preceding case, there is good agreement between the model and the data for values of $V^{1 / 3} / \sqrt{ } A_{i}>1$, corresponding to $s_{o} / b>1.5$, with an rms difference of less than $3 \%$. A more significant error occurs when $V^{1 / 3} / \sqrt{ } A_{i}<1$, where the longest dimension of the cuboid approaches the side length of the enclosure, $s_{o} / b<1.4$. The local increase in the heat transfer caused by the close proximity of these surfaces is not accounted for in the model.

\section{Cylinder in Cubical Enclosure}

The final test case involves a circular cylinder, $h_{i} / d_{i}=0.5$, in a cubical enclosure. The two-rule model formulation for $S_{\sqrt{ } A_{i}}^{\star}$ is

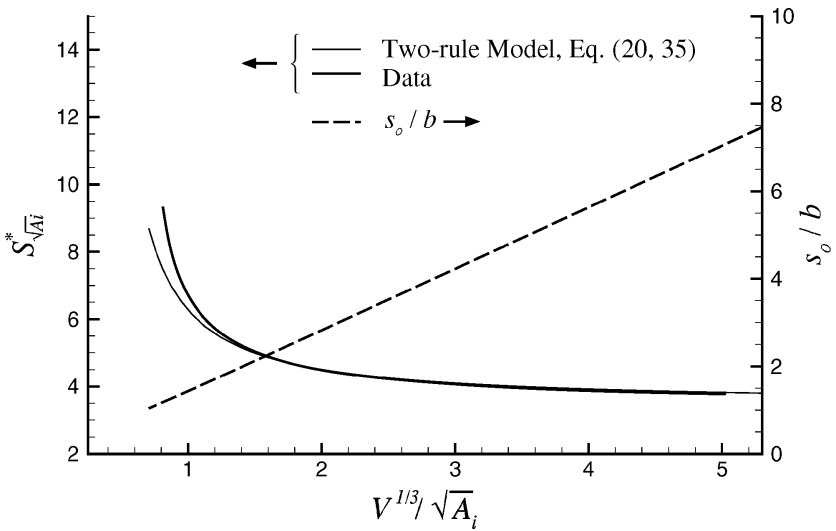

Fig. 7 Cuboid in cubical enclosure.

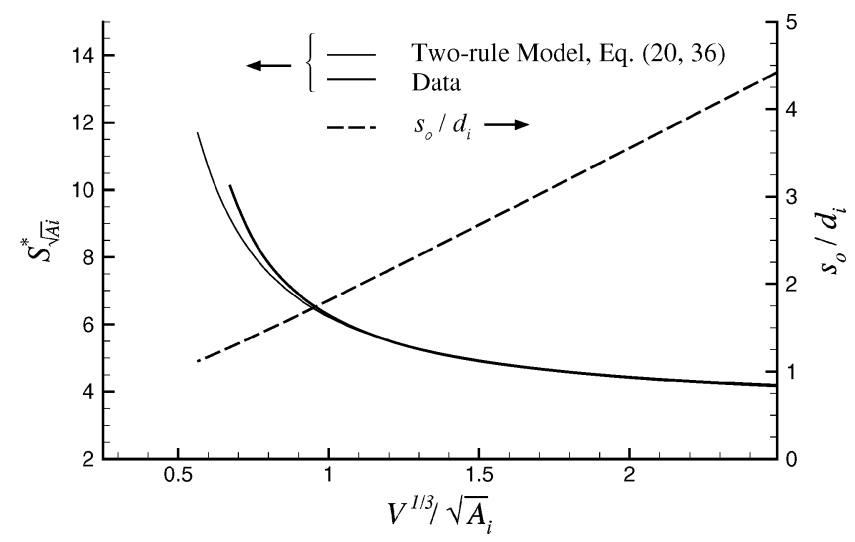

Fig. 8 Cylinder in cubical enclosure.

developed as follows:

$$
\begin{gathered}
V=s_{o}^{3}-\frac{\pi}{4} d_{i}^{2} h_{i}, \quad A_{i}=2\left(\frac{\pi}{4} d_{i}^{2}\right)+\pi d_{i} h_{i} \\
\frac{V^{\frac{1}{3}}}{\sqrt{A_{i}}}=\sqrt{\frac{2}{\pi}} \frac{\left[\left(s_{o} / d_{o}\right)^{3}-(\pi / 4)\left(h_{i} / d_{i}\right)\right]^{\frac{1}{3}}}{\sqrt{2 h_{i} / d_{i}+1}}
\end{gathered}
$$

Equation (20) is used to determine $S_{\sqrt{ } A_{i}}^{\star}$, where $S_{\infty}^{\star}$ is calculated for $h_{i} / d_{i}=0.5$ using Eq. (24).

The model predictions are validated using numerical results from simulations performed using FLOTHERM, as described in the Appendix. Figure 8 demonstrates the good agreement between the model and the data, within $2 \% \mathrm{rms}$ for $V^{1 / 3} / \sqrt{ } A_{i}>0.8$, corresponding to $s_{o} / d_{i}>1.5$. The maximum difference of $10 \%$ occurred when the diameter of the cylinder approaches the dimensions of the enclosure, $s_{o} / d_{i}=1.3$.

\section{Summary}

A general model has been presented for conduction shape factors between an arbitrarily shaped, isothermal inner body and its surrounding, isothermal enclosure based on the exact solution for the concentric spheres. The model requires an effective value of the gap spacing, and two approaches for determining $\delta_{e}$, the integral method and the two-rule method, have been developed. The integral method provides better agreement than the two-rule method with the available numerical data; however, its use is limited to geometries where the local gap thickness and integration limits can be expressed mathematically. The two-rule method is a general model, applicable in all cases where the enclosed volume and inner body surface area can be determined.

The agreement between the two-rule model and the numerical data is excellent for all enclosures with geometrically similar boundary shapes, within 1-3\% rms difference in all cases examined in this work. The two-rule model is also effective in accurately predicting 
$S^{\star}{ }_{A_{i}}$ for enclosures with different inner and outer boundary shapes, with an rms difference of $3-5 \%$ in all cases when $V^{1 / 3} / \sqrt{ } A_{i}>1$. The model is less accurate for certain geometries, such as the sphere in a cubical enclosure, and the ratio of the smallest outer body dimension to the largest inner body dimensions, that is, $s_{o} / d_{i}$, can be used to indicate the range of application of the model. In all cases, the accuracy of the two-rule model is significantly reduced when the corresponding ratio of outer and inner dimensions is less than 1.5. In these situations, numerical simulation may be required to determine accurately the conduction shape factor.

\section{Appendix: Numerical Simulations}

All numerical data for the cuboid-in-cube and cylinder-in-cube enclosures were calculated using FLOTHERM, ${ }^{7}$ a commercial finite volume-based CFD software package. The CFD modeling is performed in a three-dimensional domain, and the mode of heat transfer is limited to conduction only. The software uses an orthogonal grid in Cartesian coordinates and is well suited to problems involving cubes and cuboids; however, FLOTHERM also includes angled plates that are used to create complex objects, such as the circular cylinder.

The models for the cuboid and the cylinder are simplified by assuming one-eighth symmetry, with adiabatic boundary conditions imposed at all symmetry $(x=0, y=0$, and $z=0)$ boundaries and isothermal boundary conditions at all outer boundaries, $T_{o}=25^{\circ} \mathrm{C}$. The surface of the inner body is treated as an isothermal boundary condition, $T_{i}=50^{\circ} \mathrm{C}$. To vary the aspect ratio of the enclosure, the

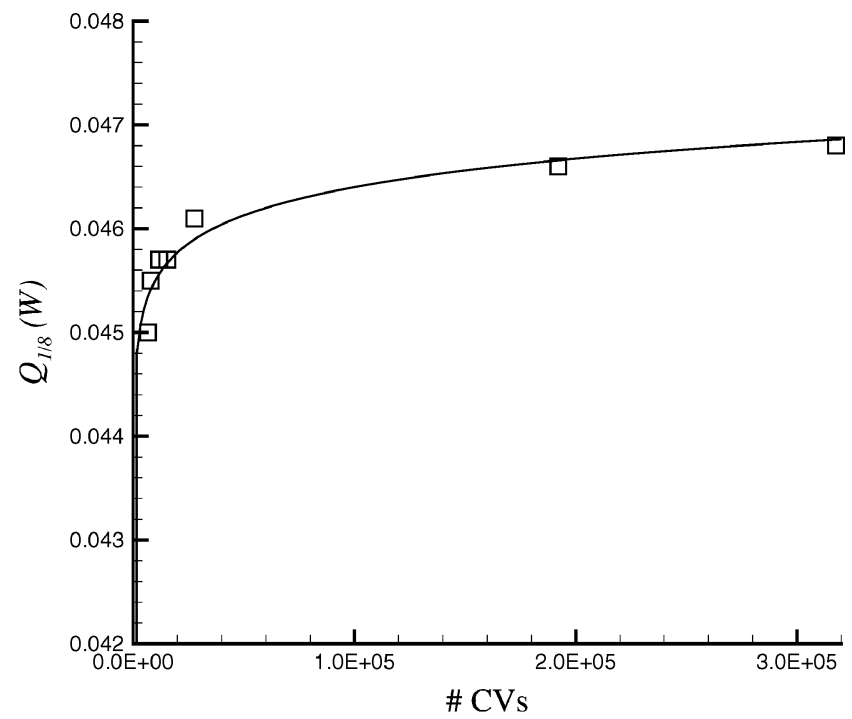

Fig. A1 Grid convergence results for cylinder in cubical enclosure. inner boundary dimensions are changed, whereas the outer boundary is held constant. A constant value for the thermal conductivity, $k=0.0261 \mathrm{~W} / \mathrm{mK}$, is specified for the region between the source and the outer boundary. The software automatically calculates the total heat flow rate crossing the inner boundary based on an area-weighted sum of the fluxes calculated from the local temperature gradient.

Grid independence was verified for the cylinder-in-cube configuration for the test case $d_{i}=60 \mathrm{~mm}, h_{i}=30 \mathrm{~mm}$, and $s_{o}=134 \mathrm{~mm}$. The total heat transfer rate is plotted as a function of the number of control volumes in Fig. A1.

The grid convergence study demonstrates that the use of approximately 200,000 control volumes for the simulation results in a solution for $Q$ that is independent of the number of grid points. All subsequent numerical simulations were performed using the same level of mesh refinement.

The results of the FLOTHERM simulations are expressed in terms of the total heat transfer rate for the one-eighth symmetry problem, $Q_{1 / 8}$. To compare these data with the model predictions, the following nondimensionalization is performed:

$$
S_{\sqrt{A_{i}}}^{\star}=8\left(Q_{1 / 8}\right) / k \sqrt{A_{i}} \Delta T
$$

where values of $k$ and $\Delta T=T_{i}-T_{o}$ are given earlier and $\sqrt{ } A_{i}$ is the square root of the total inner boundary surface area.

\section{Acknowledgments}

The authors acknowledge the continued financial support of Materials and Manufacturing Ontario and the Centre for Microelectronics Assembly and Packaging.

\section{References}

${ }^{1}$ Hassani, A. V., "An Investigation of Free Convective Heat Transfer from Bodies of Arbitrary Shape,” Ph.D. Dissertation, Dept. of Mechanical Engineering, Univ. of Waterloo, Waterloo, ON, Canada, 1987.

${ }^{2}$ Warrington, R. O., Powe, R. E., and Mussulman, R. L., "Steady Conduction in Three-Dimensional Shell," Journal of Heat Transfer, Vol. 104, May 1982, pp. 393, 394.

${ }^{3}$ Hassani, A. V., and Hollands, K. G. T., "Conduction Shape Factor for a Region of Uniform Thickness Surrounding a Three Dimensional Body of Arbitrary Shape," Journal of Heat Transfer, Vol. 112, May 1990, pp. 492-495.

${ }^{4}$ Yovanovich, M. M., "Conduction and Thermal Contact Resistances (Conductances)," Handbook of Heat Transfer, 3rd ed., edited by W. M. Rohsenow, J. P. Hartnett, and Y. Cho, McGraw-Hill, New York, 1998, Chap. 3, pp. 3.1-3.73

${ }^{5}$ Incropera, F. P., and DeWitt, D. P., Fundamentals of Heat and Mass Transfer, 4th ed., Wiley, New York, 1996, pp. 96-99.

${ }^{6}$ Culham, J. R., Yovanovich, M. M., Teertstra, P., Wang, C.-S., RefaiAhmed, G., and Tain, R., "Simplified Analytical Models for Forced Convection Heat Transfer from Cuboids of Arbitrary Shape," Journal of Electronic Packaging, Vol. 123, No. 3, 2001, pp. 182-188.

${ }^{7}$ FLOTHERM, Flomerics, Inc., Southboro, MA, 2002. 